\title{
Mediale Value-Frames - Theoretisches Konzept und methodische Herausforderungen
}

\author{
Bertram Scheufele \& Ines Engelmann
}

\begin{abstract}
Der Beitrag beschäftigt sich aus kommunikationswissenschaftlicher Perspektive mit Value-Frames bzw. Value-Framing. Er lenkt den Blick auf die Frage, welche politisch-gesellschaftlichen Werte als Bezugsrahmen an politisch-gesellschaftliche Akteure, Situationen und Probleme angelegt werden. Die Autoren arbeiten eine eigene Definition des Konstrukts heraus, die unterschiedliche Forschungsstränge aufgreift. Danach diskutieren sie das Vorgehen sowie die Vor- und Nachteile einer inhaltsanalytischen Anwendung anhand eines Forschungsprojektes zu Bundestagswahlen.
\end{abstract}

\section{Einleitung}

Der vorliegende Beitrag beschäftigt sich mit dem Konzept der Value-Frames und dabei insbesondere mit dessen inhaltsanalytischer Anwendung. Das Konstrukt des Value-Frames bzw. des Value-Framing richtet den Blick auf die Frage, welche politisch-gesellschaftlichen Werte (z. B. „Freiheit“, „Nachhaltigkeit“) als Bezugsrahmen an politisch-gesellschaftliche Akteure, Situationen und Probleme angelegt werden. Im konkreten Anwendungsfall, nämlich der kommunikationswissenschaftlichen Wahlforschung, interessiert uns zweierlei: ${ }^{1}$ (1) In den Rahmen

1 Der Beitrag greift auf theoretische Überlegungen, methodische Umsetzungen und empirische Befunde aus einem dreijährigen DFG-Projekt des Autors (SCHE 697/6-1) zurück, in dem die Autorin wissenschaftliche Mitarbeiterin war. Wir danken an dieser Stelle sowohl der Deutschen Forschungsgemeinschaft als auch unseren Codiererinnen und Codierern. 
welcher Werte stellen die Massenmedien die Parteien und deren Spitzenkandidaten bei Bundestagswahlen („Value-Framing“)? (2) Präsentieren die Massenmedien die Parteien bzw. die Spitzenkandidaten dabei als förderlich oder hinderlich für diese Werte („Instrumentalität des Value-Framing“)?

Unsere Herangehensweise ist kommunikationswissenschaftlicher Natur und damit anders gelagert als z. B. ein linguistischer Zugang. Eine gelungene Gegenüberstellung linguistischer und kommunikationswissenschaftlicher Zugänge zu Frames bietet z. B. Ziem (2013, 2014). „Während sich linguistische Frame-Ansätze für Aspekte der sprachlichen Bedeutungskonstitution interessieren, fokussiert dagegen ein kommunikationswissenschaftlich orientierter Zugang stärker auf die Untersuchung der Wirkung, Verbreitung und (strategische, massenmediale) Rahmung der Nachricht" (Ziem 2013, 144). Folglich ist das Kernkonstrukt im ersten Fall beispielsweise der an die Kasusgrammatik anknüpfende „Prädikationsrahmen" (Ziem 2013, 163), im zweiten Fall dagegen der Medien-Frame, also der z. B. in einem Zeitungsartikel oder Blogeintrag identifizierbare Bezugsrahmen. Auf die linguistische Tradition können wir hier nicht im Detail eingehen. Zumindest aber lässt sich festhalten, dass es durchaus Schnittstellen zwischen beiden Forschungstraditionen gibt, da Linguistik und Kommunikationswissenschaft auf teilweise ähnliche (Frame-)Konzepte rekurrieren. So greift z. B. Ziem (2008, 23-25) unter anderem auf Minskys (1974) Frame-Konzept zurück, das unseres Erachtens stark dem kognitionspsychologischen Schablonenmodell von Schemata (vgl. z. B. Tesser 1978, 290-291; auch Anderson 1996, 150-151) ähnelt, ${ }^{2}$ das wiederum die kommunikationswissenschaftliche Forschung aufgegriffen hat (vgl. z. B. Scheufele/Scheufele 2010, 116).

Die Kommunikationswissenschaft versteht Frames als „principles of selection, emphasis, and presentation composed of little tacit theories about what exists, what happens, and what matters" (Gitlin 1980, 6). Menschen ziehen also Bezugsoder Interpretationsrahmen - engl. Frames - heran, um Sachverhalte, Ereignisse oder Akteure einzuordnen, zu interpretieren und zu beurteilen (vgl. z. B. Entman 1993, 52; Gitlin 1980, 7-8; Goffman 1993, 19; Gamson/Modigliani 1989, 3). Die

2 So spricht auch Minsky (1974) letztlich von Slots und (Default) Values: „A conventional frame for ,story' (in general) would arrive with slots for setting, protagonists, main event, moral, etc." (Minsky 1974, 30; Herv. i. O.). Im Übrigen finden sich bei Minsky $(1974,28)$ auch explizite Bezüge auf das Script-Konzept (vgl. dazu z. B. Schank/Abelson 1977, 36-68). 
Anwendung solcher Frames etwa durch Parteien, Politiker, Interessensverbände, soziale Bewegungen oder eben auch Journalisten wird als Rahmung - engl. Framing - bezeichnet (vgl. z. B. Snow/Benford 1988, 198). Die daraus resultierenden Wirkungen auf die Rezipienten werden wiederum als Framing-Effekte bezeichnet (vgl. z. B. Price/Tewksbury 1997, 198).

Beispielsweise kann Datenschutz entweder aus der Perspektive von Sicherheit betrachtet oder in den Bezugsrahmen von Freiheit gestellt werden. Ein journalistischer Kommentar würde im ersten Fall eine Lockerung des Datenschutzes mit der Fahndung nach Kriminellen oder der Aufdeckung terroristischer Finanzierungswege rechtfertigen. Im zweiten Fall würde eine Lockerung des Datenschutzes als Einschränkung des Persönlichkeitsrechtes des Bürgers bzw. der Bürgerin gerahmt werden. Gesellschaftliche Werte wie Sicherheit und Freiheit können also Akteure als Bezugsrahmen einsetzen, um z. B. bestimmte politische Maßnahmen zu legitimieren bzw. zu unterstützen oder aber, um sie in Frage zu stellen bzw. um sie abzulehnen.

Grob lassen sich drei Forschungsbereiche zu Frames und Framing in der Kommunikationswissenschaft ausmachen (vgl. z. B. Scheufele/Scheufele 2010, 111-115):

- Erstens wird untersucht, inwiefern sich die kognitiven Bezugsrahmen bzw. Schemata von Journalisten in deren Beiträgen als Medien-Frames niederschlagen (vgl. z. B. Scheufele 2004, 403). Wenn ein Journalist an Datenschutz den Bezugsrahmen von Sicherheit anlegt, wird diese Perspektive auch in seinen Beiträgen anklingen bzw. erkennbar werden.

- Zweitens wird untersucht, inwieweit Journalisten dabei auch jene Deutungsrahmen aufgreifen, die politische und gesellschaftliche Akteure im öffentlichen Diskurs zu etablieren versuchen (vgl. z. B. Walgrave/ Manssens 2005; Johnston/Noakes 2005). „[T] he processes that influence the creation or changes of frames applied by journalists" bezeichnet D. Scheufele (1999, 115; Herv. i. O.) als Frame-Building. Beispielsweise dürften vor allem jene Politiker den Bezugsrahmen von Sicherheit bedienen, die eine verschärfte Gangart etwa bei der Vorratsdatenspeicherung durchsetzen wollen.

- Drittens wird untersucht, inwieweit Medien-Frames, die Journalisten selbst prägen oder mit denen sie die Sichtweisen z. B. politischer Akteure aufgreifen, die Vorstellungen, Urteile oder Entscheidungen ihrer Rezipienten beeinflussen können (vgl. z. B. Iyengar 1991; Price/Tewksbury 1997; 
Cappella/Jamieson 1997). Beispielsweise dürften Rezipienten einer Vorratsdatenspeicherung eher zustimmen, wenn die Medienberichterstattung Datenschutz vor allem aus der Perspektive von Sicherheit rahmt.

Bevor wir unser Konzept der Value-Frames vorstellen, sind die Perspektiven bzw. Herangehensweisen zu explizieren, die unserem Beitrag zugrunde liegen: Erstens argumentieren wir aus der Perspektive der Kommunikationswissenschaft, die als Querschnittsdisziplin auf vielfältige Anleihen aus anderen Fächern zurückgreift - so auch in Bezug auf Framing. Zweitens legen wir eine empirische Perspektive zugrunde: Wir halten also theoretische Überlegungen dann für brauchbar, wenn sie den empirischen Zugang erkennbar mitdenken bzw. ermöglichen. Drittens vertreten wir nicht einen methodischen, sondern einen problemorientierten Primat: Statt einen bestimmten methodischen Zugang von vornherein zu präferieren oder auszuschließen, halten wir es für sinnvoller, vom theoretischen Problem auszugehen und einen dafür geeigneten methodischen Zugang zu wählen. Die Vorgehensweise, die wir in diesem Beitrag vorstellen, ist daher auch nicht als methodischer ,Königsweg‘ zu verstehen, sondern als der Weg, der für unsere Fragestellung unseres Erachtens am sinnvollsten ist. Viertens legen wir eine vergleichende Perspektive an. Konkret fokussieren wir - wie zuvor schon andere Autoren (vgl. z. B. Wilke/Reinemann 2000; Sheafer/Weimann 2005) - auf mehrere Wahljahre. Auf diese Weise haben unsere Aussagen nicht nur singuläre Reichweite (z. B. für eine Wahl), sondern nähern sich dem an, was Merton (1996, 41-50; vgl. auch Opp 1970, 245) als Anspruch mittlerer Reichweite bezeichnet.

\section{Theoretische Überlegungen zum Value-Framing}

Mit dem Konzept der Value-Frames versuchen wir, eine Brücke zwischen der kommunikationswissenschaftlichen Framing-Forschung und der politikwissenschaftlichen Werteforschung zu schlagen. Den Ausgangspunkt unserer Überlegungen zu Value-Frames bilden zwei Defizite der kommunikationswissenschaftlichen Framing-Forschung. Das erste Defizit besteht darin, dass viele Studien auch eigene frühere Arbeiten - sehr spezifische, nur auf ein Einzelthema zugeschnittene Frames betrachten (vgl. auch z. B. Benford 1997, 414; de Vreese et al. 2001, 108; Matthes 2007, 79-80). Abstrakte, themenunabhängige Frames, die z. B. de Vreese (2010, 189; Herv. i. O.) „generic news frames“ nennt, werden dagegen 
seltener untersucht. Damit verbunden ist das zweite Defizit: Die bisherigen Vorschläge für themenübergreifende Frames (vgl. z. B. Semetko/Valkenburg 2000; Dahinden 2006, v. a. 105-109; Valkenburg et al. 1999) lassen sich im Grunde auf eine einzige Untersuchung von Neuman et al. (1992, 74-76) zurückführen, die „conflict“, „moral values“, „economics“, „powerlessness“ und „human impact“ als abstrakte Frames vorschlagen. Allerdings sind diese Frames theoretisch nicht fundiert (vgl. Scheufele 2010, 27) und zudem eher Nachrichtenfaktoren („conflict“, „human impact“) oder Themen („economics“) (vgl. Scheufele 2004, 43).

Ein überzeugenderer Vorschlag für themenübergreifende Frames ist möglich durch Rückgriff auf das Konzept der Value-Frames, das bislang vor allem in der experimentellen Medienwirkungsforschung beheimatet ist (vgl. dazu z. B. Shah et al. 1996; Nelson et al. 1997b; Domke et al. 1998; Brewer 2002; Brewer/Gross 2005; Shen/Edwards 2005; Scheufele 2010). Solche Studien zu Value-Framing-Effekten (vgl. im Überblick Shah et al. 2001, 229-230; Scheufele 2010, 27-30), die stark von der Kognitionspsychologie und der Persuasionsforschung geprägt sind, schlagen aber eher selten einen expliziten Bogen zur politikwissenschaftlichen Werteforschung (vgl. z. B. Inglehart 1977; Klingemann/Volkens 2002; Fuchs 1991). Wenn das Konstrukt des Value-Frames aber als Vorschlag für themenübergreifende Rahmen überzeugen soll, ist es dezidierter an solche Wertekonzepte anzubinden. Allgemein gelten Werte als „abstrakte Entwürfe sozial erstrebenswerter Zustände, die als präskriptive Aussagen in moralischen Diskursen aufscheinen“ (Klein 2005, 424). Denkbar ist nun zwar der Rückgriff auf sozialpsychologische Wertekonzepte (vgl. z. B. Schwartz/Bardi 2001). Für unsere Fragestellung waren aber politikwissenschaftliche Überlegungen relevanter. Politische Werte lassen sich definieren als die

von den Mitgliedern einer politischen Gemeinschaft als gemeinsam anerkannten Vorstellungen von den anzustrebenden Zielen des politischen Zusammenlebens und den zur Erreichung dieser Ziele angemessenen Mitteln. (Gabriel 2009, 31)

Folglich sind politische Werte als Teil der politischen Kultur zu sehen (vgl. z. B. Verba 1965, 513). Mit diesen Überlegungen sprechen wir von Value-Frames,

wenn politische Werte bzw. Prinzipien, die in der politischen Kultur sedimentiert sind, als Bezugsrahmen für übergeordnete Politikfelder, politische Akteure und Vorgänge fungieren. (Scheufele/Engelmann 2012, 35; Herv. i. O.) 
Diese Nominaldefinition beinhaltet fünf zentrale Aspekte, die weiter auszuführen sind:

- Abstraktionsniveau

- Sedimentierung

- Stabilität

- Cleavages und Wertekonflikte

- Instrumentalität und Bewertung

Value-Frames sind erstens auf einem höheren „Abstraktionsniveau“ anzusiedeln als konkrete, themenspezifische Frames. Ein höheres Abstraktionsniveau postulieren auch andere Autoren für Meta-Botschaften (vgl. z. B. Vlasic 2004, 205-211) oder für Master-Frames (vgl. z. B. Snow/Benford 1992; Ayers 2004). So sprechen Benford und Snow $(2000,619)$ nur dann von einem Master-Frame, wenn ein solcher Rahmen von mindestens zwei unterschiedlichen sozialen Bewegungen aufgegriffen wird. Vergleichbar dazu lassen sich Werte auf ganz unterschiedliche Themen oder Politikfelder anwenden. Beispielsweise kann eine Partei bei verschiedenen Themen in den Rahmen von Freiheit oder von Sicherheit gestellt werden. Somit sind Werte dafür prädestiniert, als Zentralkategorie für abstrakte Frames - also die besagten Value-Frames - zu dienen.

Zweitens können Werte nur dann als Bezugsrahmen wirksam werden, wenn sie in der politischen Kultur ${ }^{3}$ hinreichend „sedimentiert“ sind. Damit knüpfen wir an politikwissenschaftliche und soziologische Überlegungen zu Werten und Kultur an, wonach Werte den Kern der politischen bzw. gesellschaftlichen Kultur bilden (vgl. z. B. Fuchs 2002, 36), weil die Bürger Werte im Sozialisationsprozess internalisiert haben (vgl. dazu z. B. Parsons 1951, 207-226; Habermas 1981/1995, 217; Mahrt 2010, 36-41). So wissen Bürger grob, was Freiheit oder Gerechtigkeit bedeuten - auch wenn jede/r sicher eine etwas andere Vorstellung von Freiheit oder Gerechtigkeit haben mag.

Eng verbunden mit dem Merkmal der Sedimentierung ist drittens der Aspekt der „Stabilität“ von Value-Frames, was freilich nicht absolute Unveränderlichkeit

3 Unter politischer Kultur versteht Schulz $(2011,45)$ „das spezifische Muster individueller Einstellungen und Orientierungen der Systemmitglieder gegenüber Politik. Sie besteht aus mehreren Komponenten: (1) aus politischen Kenntnissen und Überzeugungen, (2) aus der affektiven Beziehung zu politischen Akteuren, also z. B. Parteien oder Politikern, und (3) aus Urteilen und Meinungen". Ähnlich definiert Verba $(1965,513)$ politische Kultur als „the system of empirical beliefs, expressive symbols and values which defines the situation in which political action takes place“. 
bedeutet. Busse und Teubert (1994, 24; Herv. i. O.) betonen zu Recht, dass Diskurse „schon vom Begriff her eine diachrone Größe“ sind. Somit sind auch Frames, die in Diskursen prozessiert werden, nicht unveränderlich. Es wäre auch erschreckend, wenn sich z. B. die Bedeutung von Freiheit seit dem Mittelalter nicht verändert hätte. Allerdings hält Matthes (2007, 82) der kommunikationswissenschaftlichen Framing-Forschung vor, nur selten nach Frame-Veränderungen zu fragen. Wenn überhaupt werde meist nur „die Zunahme oder Abnahme von fest stehenden Frames“ betrachtet. Diese Einschätzung ist einerseits nicht ganz falsch, offenbart aber andererseits ein zu enges Verständnis von semantischem Wandel bzw. eine empirisch nur bedingt anzutreffende Form des gesellschaftlichen Wandels. Tatsächlich zeigt die Werteforschung, dass sich in den letzten Dekaden gar nicht so sehr die Bedeutung einzelner Werte verändert hat, sondern sich vielmehr die Gewichte von Werten verschoben haben. Beispielsweise fand eine Verlagerung von materialistischen Werten (z. B. wirtschaftlicher Wohlstand) auf postmaterialistische Werte (z. B. Selbstverwirklichung, politische Partizipation) statt (vgl. z. B. Inglehart 1977; Klingemann/Volkens 2002; Fuchs 1991; Hildebrandt/ Dalton 1977, 236-242; Klein 2005, 434-441) - ohne dass sich die Bedeutung dieser Werte substanziell verändert hätte. In vergleichbarer Weise kann sich das Gewicht von Frames im Zeitverlauf verändern, wobei dann alte und neue Frames nebeneinander in Diskursen präsent sein können. Ähnlich argumentieren auch Busse und Teubert $(1994,24)$ in Bezug auf Sprachwandel (vgl. auch z. B. Harden 2002, 204-207). Nach einer Zu- und Abnahme bestehender Frames zu fragen, ist also keineswegs unzulässig - im Gegenteil. Darüber hinaus müssen Frames natürlich einigermaßen beständig sein, um überhaupt als Orientierungsanker dienen zu können. Denn was sich ständig in diversen Details ändert, kann gar keine Orientierung bieten. Obwohl es also immer auch gewisse Abweichungen und „Modulationen“ (Goffman 1993, 52) von Frames geben wird, ist für uns die Frage, wie sich die Gewichtung themenübergreifender Frames im Zeitverlauf verlagert, relevanter als die Frage nach der Veränderung des Sinngehalts einzelner, thematisch beschränkter Frames. Im Übrigen zeichnen Matthes und Kohring (2004) mittels Clusteranalyse gar keine Veränderungen von Frames, sondern lediglich eine Veränderung der journalistischen Kombination von Frames, also eine Verände- 
rung journalistischer Framing-Strategien nach - das ist ein gewichtiger Unterschied zum Frame-Wandel (vgl. dazu Scheufele/Scheufele 2010, 122; Scheufele/Engelmann 2012, 179).

Viertens rekurrieren wir auf politikwissenschaftliche Arbeiten zu „gesellschaftlichen Cleavages" (vgl. z. B. Lipset/Rokkan 1967; Pappi/Shikano 2002). Lipset und Rokkan (1967, 2-3; Herv. i. O.) sprechen von „historically given ,packages' of programs, commitments, outlooks, and, sometimes, ,Weltanschauungen “", um die herum sich die Bürger in sozialstrukturellen „konfligierenden Wertgemeinschaften" (Fuchs 1991, 69) gruppieren und sich auch die Parteien entsprechend positionieren (vgl. Lipset/Rokkan 1967, 26, 50). Cleavages sind also mehr oder minder stark "in der Sozialstruktur einer Gesellschaft verankerte“ (Klein 2005, 427) Wertekonflikte ${ }^{4}$. Am häufigsten diskutiert wird in der jüngeren Forschung wohl Ingleharts (1977) These des Wandels von materialistischen zu postmaterialistischen Werten. In einem späteren Beitrag spricht Inglehart (1988) von „Modernisierung“ und „Postmodernisierung“. Damit meint er die mit dem ersten Konflikt vergleichbare Verschiebung von der Frage des „Überlebens“ zur Frage des „Wohlbefindens“ (Inglehart 1988, 122). Offenbar ist diese Sichtweise aber zu einfach. So zielt Fuchs (1991) auf den Konflikt zwischen links- und rechtsmaterialistischen Werten, der auf den „alten Klassengegensatz“ (Klein 2005, 435) zwischen egalitärer Verteilung des Wohlstandes und Verteilung nach dem Leistungsprinzip abzielt. Ein weiteres Cleavage wird zwischen autoritären und libertären Werten gesehen (vgl. z. B. Flanagan/Lee 2003, 235-238), wobei dieser Wertegegensatz laut Klein $(2005,436)$ keine sozial-strukturelle Entsprechung hat. Analog spricht Niedermayer $(2003,11)$ nur von einer politisch-kulturellen Konfliktlinie. Das Aufkommen libertärer Positionen verbindet er vor allem mit dem Einzug der Grünen in den Bundestag bei der Bundestagswahl 1983 (vgl. Niedermayer 2003, 24). Stöss (1997, 163; Herv. i. O.) sieht die „Hauptachse der Parteienkonkurrenz [... mittlerweile] zwischen einer sozial-libertären und einer neoliberal-autoritären Politikkonzeption“. Der letzte Aspekt unserer Nominaldefinition betrifft die „Instrumentalität" von Value-Frames. ${ }^{5}$ Entman $(1993,52)$ konzipiert Bewertungen

4 Lipset/Rokkan $(1967,47)$ führen vier zentrale Konfliktlinien an: „Center-Periphery“, „StateChurch“, „Land-Industry“ und „Owner-Worker“.

5 Entman (2007, 165, Herv. i. O.) hat zudem mit „news slant“ einen neuen Begriff geprägt, der aber noch mehr Verwirrung in der Frage nach Frames und Bewertungen stiftet. Im Gefolge Entmans $(1993,2007)$ argumentiert z. B. auch Matthes (2007, 136-137, 140). Wir richten uns wohlgemerkt 
als Element von Frames. Tatsächlich sind beide Konstrukte - Frames und Bewertungen - aber sauber zu trennen. Bereits Gamson und Modigliani $(1989,4)$ betonen: „Frames should not be confused with positions for or against some policy measure“ (vgl. ähnlich Tankard 2001, 96).

Bei Framing geht es also gerade nicht darum, Positionen, Urteile oder Bewertungen explizit auszusprechen, sondern zunächst nur um die Rahmung an sich. Folgendes Beispiel illustriert das leicht nachvollziehbar: Wenn die eine Zeitung die SPD als Garant von Solidarität betrachtet, eine andere Zeitung die SPD dagegen als Gefahr für Solidarität sieht, dann legen beide Zeitungen den gleichen wertgebundenen Bezugsrahmen der Solidarität an. Freilich beurteilen sie die SPD unterschiedlich in der Frage, ob die Partei für Solidarität förderlich oder hinderlich ist. Mit dieser Überlegung rekurrieren wir auf Rosenbergs (1956) Ansatz kognitiv-affektiver Konsistenz, wonach die Einstellung zu einem Objekt umso positiver bzw. negativer ist, je förderlicher bzw. hinderlicher das Objekt für zentrale Werte ist. Wer die SPD als förderlich für Solidarität, Frieden und ökologische Nachhaltigkeit ansieht (positive Instrumentalität), wird diese Partei positiv bewerten (positive Gesamteinstellung). Wer sie dagegen als hinderlich oder gefährlich für diese Werte betrachtet (negative Instrumentalität), wird eine negative Haltung zur SPD haben (negative Gesamteinstellung). Auf ähnliche Weise gehen Fishbein und Ajzen (1975, 30-32, 222-235; vgl. auch Ajzen 2005, 123-124) davon aus, dass Menschen von Objekten bestimmte Merkmale („attribute“) erwarten, wobei mit jedem Attribut zugleich eine entsprechende Bewertung („evaluation“) assoziiert wird. Die Gesamteinstellung eines Menschen zum betreffenden Objekt ergibt sich

by multiplying his evaluation of each attribute associated with the object by his subjective probability that the object has that attribute and then summing the products. (Fishbein/Ajzen 1975, 223)

Darauf aufbauend differenzieren Nelson et al. (1997a, 226) deutlich zwischen Persuasion und Framing: Einstellungsänderung könne ein „change of the individual's beliefs or cognitions about the attitude object (traditional persuasion) or change how the individual weights that information (framing)“ sein. Zusammen-

nicht gegen die anderen Frame-Elemente bei Entman (1993), sondern nur dagegen, Bewertungen als Frame-Element aufzunehmen. 
fassend lässt sich also festhalten: Frames und Bewertungen sind zwei unterschiedliche Konstrukte. Mit Rosenberg (1956) lassen sich beide sowohl trennscharf unterscheiden als auch in sinnvolle Beziehung zueinander setzen - mit dem obigen Beispiel lässt sich dies wie folgt illustrieren: ${ }^{6}$ Wenn eine Zeitung die SPD als Gefahr für Solidarität rahmt, die andere Zeitung die SPD als Garant für Solidarität darstellt, dann liegt identisches Value-Framing, aber unterschiedliche Instrumentalität vor. Wenn eine Zeitung die SPD als Gefahr für Solidarität rahmt und die andere Zeitung die SPD als Gefahr für Freiheit sieht, dann liegt identische Instrumentalität, aber unterschiedliches Value-Framing vor.

Ausgehend von den dargelegten theoretischen Überlegungen haben wir in unserem Wahlforschungsprojekt unter anderem die beiden folgenden Forschungsfragen gestellt:

- Value-Framing: Welche politisch-gesellschaftlichen Werte legten die Qualitäts- und die Boulevardpresse als Bezugsrahmen (Value-Frames) an die CDU/CSU und die SPD sowie deren Spitzenkandidaten bei Bundestagswahlen an?

- Instrumentalität: Präsentieren die Qualitäts- und die Boulevardpresse diese Parteien und Kandidaten dabei als Garant oder als Gefahr für diese Werte?

Mit diesen Forschungsfragen stellt sich zugleich eine Reihe methodischer Fragen und Herausforderungen: Wir skizzieren dazu zunächst unsere Untersuchungsanlage und gehen dann ausführlicher auf die Frage ein, wie sich Value-Frames identifizieren lassen. Danach folgen einige ausgewählte Befunde. Im abschließenden Abschnitt diskutieren wir schließlich, welche methodischen Implikationen der von uns gewählte methodische Zugang mit sich bringt.

6 Unsere Argumentation ist auch mit der Prospect Theory kompatibel, wonach Framing nur eine Variation in der Formulierung und nicht in der expliziten Bewertung meint. Im berühmten AsianDisease-Beispiel wird eine medizinische Maßnahme in der einen Experimentalgruppe unter dem Gesichtspunkt des Sterbens („mortality frame“) und in der anderen Experimentalgruppe aus der Perspektive des Überlebens („survival frame“) gerahmt (vgl. z. B. Tversky/Kahneman 1986, 254). Eine Bewertung wird auf diese Weise zwar nahe gelegt, ist aber nicht Teil oder Element des Frames - schon weil sie gar nicht experimentell induziert wurde. 


\section{Methodische Überlegungen zur Erfassung medialer Value-Frames}

Die Untersuchungsanlage unserer Inhaltsanalyse lässt sich wie folgt skizzieren (vgl. ausführlich Scheufele/Engelmann 2012, 2013): Untersucht wurde die Vorwahlberichterstattung der fünf überregionalen Qualitätszeitungen ,Frankfurter Allgemeine Zeitung' (FAZ), ,Süddeutsche Zeitung، (SZ), ,Frankfurter Rundschau“ (FR), ,Die Welt' (WELT) und , die tageszeitung' (TAZ) sowie des Boulevardblatts ,Bild‘ (BILD). Die Qualitätszeitungen decken das Spektrum politisch-redaktioneller Linien ab und gelten als Meinungsführermedien mit breitem, journalistisch anspruchsvollem Angebot (vgl. z. B. Eilders 2004a, 144-145; Wilke 1999, 302-303, 310-312; auch Reinemann 2003; Eilders 2004b). Die BILD steht dagegen für reichweitenstarken Boulevardjournalismus, der sich auch an weniger gebildete Rezipienten wendet (vgl. z. B. Semetko/Schoenbach 2003, 55-56; auch Schirmer 2001; Reinemann 2008). Zudem gilt das Blatt „als Sprachrohr des kleinen Mannes auf der Straße“ (Dulinski 2006, 29). Mit der eingangs erwähnten wahlvergleichenden Perspektive berücksichtigten wir die vier Bundestagswahlen 1976, 1983, 1994 und 2002, wobei der jeweilige Untersuchungszeitraum die acht Wochen vor dem betreffenden Wahltermin umfasste. Diese Wahljahre wurden mit Blick auf Werteorientierungen, aber auch aus forschungspraktischen Gründen ausgewählt (vgl. ausführlich Scheufele/Engelmann 2012, 125-130). Die acht Wochen können „als ,heiße Wahlkampfphase“ bezeichnet“ (Lieske 2005, 101) werden.

Die Codiereinheit auf der ersten Ebene war der einzelne Beitrag, für den neben den formalen Kategorien bis zu vier Bezugsobjekte codiert werden konnten. Mit dem Konzept des Bezugsobjekts lehnen wir uns an eine Modifikation sozialpsychologischer Modelle an, bei der Brettschneider (2002, 211-213) zwischen Bezugsobjekten und Bewertungsdimensionen differenziert. Der zu codierende Beitrag konnte über folgende „Bezugsobjekte“ informieren: die CDU/CSU, die SPD und/oder deren jeweilige Kanzlerkandidaten. Pro Bezugsobjekt - als Codiereinheit auf der zweiten Ebene - konnten bis zu drei Themen bzw. Politikfelder sowie die zentralen Kandidaten-/Parteibezüge erfasst werden. Beispielsweise konnte der Beitrag die Kompetenz (Kandidatenbezug) von Kanzler Schröder (Bezugsobjekt) in der Sozialpolitik (Thema/Politikfeld) thematisieren. Die Kombination aus

Thema bzw. Politikfeld und Kandidaten- bzw. Parteibezug bildete die einzelne 
„Information“. Pro Information - als Codiereinheit auf der dritten Ebene - konnten bis zu zwei „Value-Frames“ codiert werden. Beispielsweise konnte die Information über Schröders Kompetenz in der Sozialpolitik in den Bezugsrahmen von Solidarität gestellt werden. Für jeden Value-Frame wurde zusätzlich die „Instrumentalität" codiert, wobei der Wertebereich von -1 (,Gefahr') bis +1 (,Garant' für den betreffenden Wert) reichte.

\begin{tabular}{|c|c|c|c|c|}
\hline \multirow[b]{2}{*}{$\begin{array}{l}\text { Grundausrich- } \\
\text { tung }\end{array}$} & \multicolumn{2}{|c|}{ Quantitativer Zugang } & \multicolumn{2}{|c|}{ Qualitativer Zugang } \\
\hline & $\begin{array}{l}\text { Induktiv-ex- } \\
\text { plorativ }\end{array}$ & Deduktiv & $\begin{array}{l}\text { Induktiv-inter- } \\
\text { pretativ }\end{array}$ & $\begin{array}{l}\text { Induktiv-explo- } \\
\text { rativ }\end{array}$ \\
\hline $\begin{array}{l}\text { Erhebungsver- } \\
\text { fahren }\end{array}$ & $\begin{array}{l}\text { Quantitative } \\
\text { Inhaltsanalyse }\end{array}$ & $\begin{array}{l}\text { Quantitative } \\
\text { Inhaltsanalyse }\end{array}$ & $\begin{array}{l}\text { Interpretative } \\
\text { bzw. herme- } \\
\text { neutische Ver- } \\
\text { fahren }\end{array}$ & $\begin{array}{l}\text { Qualitative } \\
\text { Inhaltsanalyse }\end{array}$ \\
\hline $\begin{array}{l}\text { Kategorien } \\
\text { (Codiert } \\
\text { werden ...) }\end{array}$ & $\begin{array}{l}\text { Frame-Ele- } \\
\text { mente bzw. } \\
\text { Frame-Indika- } \\
\text { toren }\end{array}$ & $\begin{array}{l}\text { Vorab defi- } \\
\text { nierte Frames }\end{array}$ & $\begin{array}{l}\text { Frame-Ele- } \\
\text { mente bzw. } \\
\text { Frame-Indika- } \\
\text { toren }\end{array}$ & $\begin{array}{l}\text { Frame-Ele- } \\
\text { mente bzw. } \\
\text { Frame-Indika- } \\
\text { toren }\end{array}$ \\
\hline $\begin{array}{l}\text { Generierung } \\
\text { bzw. Identifi- } \\
\text { zierung von } \\
\text { Medien-Fra- } \\
\text { mes }\end{array}$ & $\begin{array}{l}\text { Synthetisie- } \\
\text { rung von Fra- } \\
\text { mes z. B. über } \\
\text { Cluster- oder } \\
\text { Faktorenana- } \\
\text { lyse }\end{array}$ & $\begin{array}{l}\text { Theoriegelei- } \\
\text { tete Definition } \\
\text { von Frames } \\
\text { oder Rückgriff } \\
\text { auf bekannte } \\
\text { Frames }\end{array}$ & $\begin{array}{l}\text { Interpretativ- } \\
\text { hermeneuti- } \\
\text { sche Identifi- } \\
\text { zierung von } \\
\text { Frames aus } \\
\text { dem Material }\end{array}$ & $\begin{array}{l}\text { Identifizierung } \\
\text { von Frames } \\
\text { z. B. mittels } \\
\text { typisierender } \\
\text { Strukturierung }\end{array}$ \\
\hline
\end{tabular}

Tabelle 1: Methodische Zugänge zu Medien-Frames (vgl. Scheufele / Engelmann 2012, 175)

Die zentrale methodische Entscheidung unserer Untersuchung betraf die Frage, auf welche Weise sich Value-Frames am besten identifizieren und codieren lassen. Basierend auf einer älteren eigenen Klassifikation unterscheiden wir quantitative und qualitative Zugänge der Frame-Identifikation (vgl. Tabelle 1):

- Quantitative Zugänge: Bei einer ersten Variante werden Indikatoren für Frames oder für Frame-Elemente erfasst. Anschließend werden Beiträge anhand dieser Indikatoren mittels Cluster-Analyse gruppiert (vgl. z. B. Matthes/Kohring 2004) oder die Indikatoren werden mittels Faktorenanalyse verdichtet (vgl. z. B. Harden 2002). Bei der zweiten Variante werden 
vordefinierte, also gleichsam fertige Medien-Frames codiert, die entweder theoretisch begründet oder aus anderen Studien übernommen werden. ${ }^{7}$

- Qualitative Zugänge nutzen weder bei den Erhebungs- noch bei den Auswertungsschritten quantifizierend-statistische Verfahren. Manche Studien beschreiten hermeneutische bzw. interpretative Wege (vgl. z. B. Fair/ Astroff 1991, u. a. 66-68), andere wenden beispielsweise die Techniken der qualitativen Inhaltsanalyse nach Mayring (2000) an. Die auf diesem Wege ermittelten Frames können wiederum im Kategoriensystem quantitativer Inhaltsanalysen eingesetzt werden (vgl. z. B. Harden 2002).

Teilweise ähnlich unterscheiden Matthes und Kohring $(2004,61)$ zwischen textwissenschaftlichem Ansatz, interpretativ-quantifizierendem Ansatz, Frame-Mapping und deduktiver Frame-Analyse. Die Autoren codieren Frame-Elemente nach Entman (1993) und verdichten diese clusteranalytisch. Das entspricht dem ersten methodischen Zugang in Tabelle 1, der - wie auch der vierte methodische Zugang - eher induktiv-explorativ vorgeht. Zweifellos sind beide Zugänge ihrerseits nicht voraussetzungslos und daher nicht ausschließlich induktiv. Beide Vorgehensweisen basieren aber auf kleineren Bedeutungseinheiten (Paraphrasen, Indikatoren, Frame-Elementen o. ä.), die auf explorative Weise zu größeren Bedeutungseinheiten (Clustern bzw. ,Frames') verdichtet werden. Der zentrale Unterschied besteht im Standardisierungsgrad dieser Verdichtung: Während z. B. Matthes und Kohring (2004) ein clusteranalytisches, also statistisches Verfahren wählen, nutzen z. B. Beck und Vowe (1995) die von Mayring (2000) vorgeschlagenen qualitativen Techniken der Strukturierung (z. B. Typenbildung). Der dritte Zugang in Tabelle 1, die interpretativ-hermeneutische Identifizierung von Frames aus dem Material, ist schon aus methodologischen Gründen für unser Forschungsproblem weniger brauchbar.

Zur Beantwortung unserer Forschungsfragen haben wir uns für eine deduktivtheoriegeleitete Kategorienbildung von Werten bzw. Value-Frames entschlossen. Das entspricht dem zweiten methodischen Zugang in Tabelle 1. Um relevante politisch-gesellschaftliche Werte zu identifizieren und zu definieren, die als Ausprägungen für die Kategorie ,Value-Frame‘ in Frage kommen, wurde auf vier Typen von Quellen zurückgegriffen:

7 Hier sprechen Zeller et al. $(2010,507)$ von „holistischen Frames“. 
- Forschung zu Cleavages, Werteorientierungen und Ideologien

- Analysen von Partei- und Wahlprogrammen

- Politikwissenschaftliche Lexika und Handbücher

- Codebücher vergleichbarer Studien

Die erste Quelle bildete die politikwissenschaftliche Forschung zu Cleavages, Werteorientierungen und Ideologien (vgl. z. B. Lipset/Rokkan 1967; Fuchs 1991; Pappi/Shikano 2002, 2004; Roller 2002; Klein 2005). Sie gibt Aufschluss über zentrale Wertorientierungen und deren Wandel. Die zweite Quelle waren politikwissenschaftliche Analysen von Partei- und Wahlprogrammen (vgl. z. B. Volkens 1996, 2002; Fuchs/Klingemann 1989; Klingemann 1989; Klingemann/Volkens 2002; Pappi/Shikano 2004). Sie geben Auskunft, für welche Werte sich die beiden größeren Parteien, aber auch die kleineren Parteien stark machen und was sie mit diesen Werten jeweils verbinden. Als dritte Quelle dienten uns politikwissenschaftliche Lexika und Handbücher (vgl. u. a. Andersen/Woyke 2003; Göhler et al. 2006; Holtmann et al. 1991; Nohlen/Schultze 2005a, b; Schubert/Klein 2006). Sie dienten dazu, den Bedeutungsgehalt der betreffenden Werte weiter zu konkretisieren. Als vierte Quelle fungierten Codebücher inhaltsanalytischer Studien, die eine ähnliche Fragestellung verfolgten wie unser Projekt. Dabei orientieren wir uns nicht nur bei der Codierung von Werten, sondern auch bei der Codierung von Politikfeldern an den Codebüchern von Eilders und Lüter (1998), Volkens (2002), Wüst und Volkens (2003) sowie an den Vorschlägen von Voltmer (1997) und Klingemann/Volkens (2002, 516), an die wiederum Eilders (2004a) anknüpft. Allerdings gehen wir vor allem in zwei zentralen Punkten anders vor: Zum einen zielt unsere Inhaltsanalyse nicht auf politische Positionen, sondern auf einzelne Werte, die in der Berichterstattung als Bezugsrahmen für Parteien und Kandidaten fungierten. Zum anderen haben z. B. Voltmer (1997) oder Eilders (2004a) Werte als bipolare Konfliktstruktur erfasst. Wir codieren dagegen einzelne Werte, denn mehrere Autoren (vgl. z. B. Fuchs 1991; Stöss 1997) geben den Gedanken bipolarer Konfliktstrukturen auf. Das ist auch naheliegend, da viele Werte (z. B. Freiheit) nicht nur einzigen Wert (z. B. Sicherheit), sondern meist mehrere Werte (z. B. Sicherheit, Solidarität) als Pendant haben (vgl. z. B. Roller 2000, 94). 


\section{Vorteile und Nachteile des Ansatzes zur Erfassung medialer Value-Frames}

Unser methodischer Zugang zu medialen Value-Frames hat Vorteile, aber auch Beschränkungen bzw. Nachteile. Zu den Vorteilen gehören:

- Validität

- Anschlussfähigkeit an Politikwissenschaft

- Empirische Tragfähigkeit und Flexibilität

- Trennschärfe zwischen Framing und Bewertung

Abgesehen davon, dass synthetische Verfahren nicht immer trennscharfe Frames ermitteln (vgl. z. B. bei Matthes/Kohring 2004, 68-69), sondern eher journalistische Framing-Strategien identifizieren, dürfte es sich als schwierig erweisen, über solche Verfahren Value-Frames zu identifizieren. Frame-Elemente nach Entman (1993) zu codieren, gewährleistet ebenso wenig eine valide Erfassung von ValueFrames wie die Codierung etwa von Schlüsselwörtern. Beispielsweise zielt die Aussage „Es ist ungerecht, dass Frauen gegenüber Männern benachteiligt werden“ gar nicht auf Gerechtigkeit, sondern auf den Gleichheitsgrundsatz, selbst wenn man diese Benachteiligung ungerecht findet. Umgekehrt dient mancher Ruf nach „Gleichstellung“ vielleicht eher der Durchsetzung individueller Interessen und weniger der Durchsetzung einer allgemeinen Gleichbehandlung der Geschlechter. Wenn man also nach der Validität der Erfassung von Value-Frames fragt, dürfte die Codierung vorab definierter Value-Frames gegenüber einer Codierung von Frame-Elementen bzw. -Indikatoren der Vorzug zu geben sein.

Unser Vorschlag zur Erfassung von Value-Frames ist deswegen auch anschlussfähig an politikwissenschaftliche Überlegungen und Befunde, weil der theoretische Rückgriff auf die vier genannten Quellen bzw. Grundlagen zur Identifizierung von Werten bzw. Value-Frames gewährleistet, dass wir nicht unsere eigenen Maßstäbe anlegen, sondern jene Werte, die erstens tatsächlich in der politischen Kultur sedimentiert sind, die zweitens von den Parteien selbst in ihren Wahl- bzw. Parteiprogrammen als Bezugsrahmen aufgespannt werden und über deren Bedeutungsgehalt drittens in der Politikwissenschaft ein gewisser Konsens besteht.

Darüber hinaus zeichnet sich unser Vorgehen durch empirische Tragfähigkeit und Flexibilität aus. Die Codierung einzelner Value-Frames ermöglicht, die in der 
Vorwahlberichterstattung identifizierten Wertebezüge auf flexible Weise zu unterschiedlichen Ideologien oder Wertehorizonten (z. B. autoritäre vs. libertäre Werte) zu bündeln. Zudem lässt sich mit unserer Vorgehensweise die - schon in theoretischer Hinsicht zentrale - Instrumentalität des Value-Framing angemessen erfassen. Die empirische Tragfähigkeit zeigt sich im konkreten Anwendungsfall, wobei wir nur für unsere eigene Studie sprechen können - darauf werden wir noch zurückkommen. An dieser Stelle genügen exemplarische Befunde.

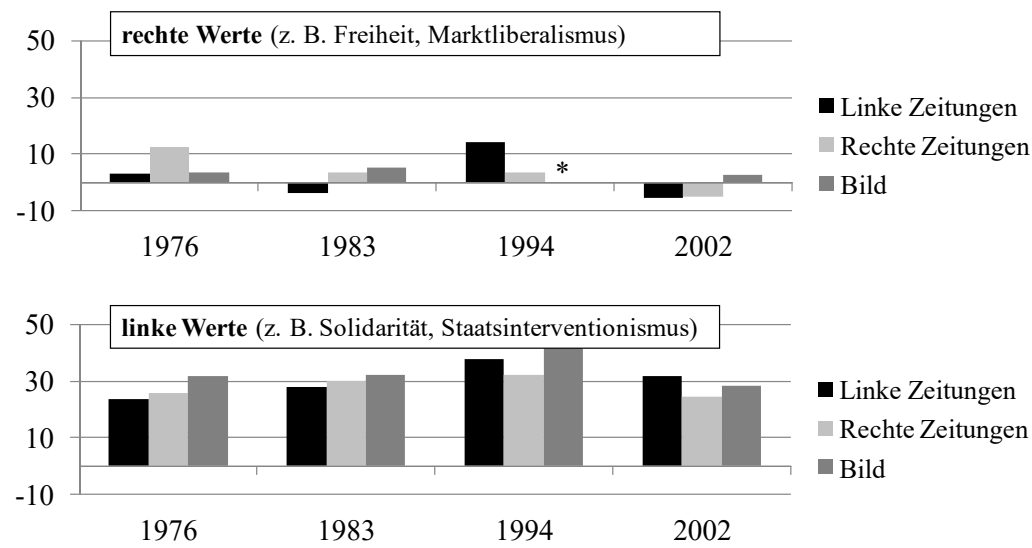

Abb. 1: Publizistische Werte-Positionierung der SPD und ihrer Kandidaten für linke und rechte Werte $\left({ }^{*} \mathrm{n}<5\right)$

Abbildung 1 und 2 zeigen die publizistische Werte-Positionierung der CDU/CSU und der SPD. Als publizistische Werte-Positionierung bezeichnen wir das Produkt aus dem Anteil der wertbezogenen Rahmung einer Partei und der Instrumentalität dieses Value-Framing. Beispielsweise kann die FAZ in einem Wahljahr die CDU/CSU in $18 \%$ aller Fälle ${ }^{8}$ in den Rahmen von Freiheit stellen und die Partei dabei als erkennbaren Garant für Freiheit (Mittelwert $+0,8$ ) präsentieren. ${ }^{9}$ Multipliziert man den Anteil dieser Rahmung (18\%) und die betreffende Instrumentalität $(+0,8)$, so ergibt sich für Freiheit eine publizistische Werte-Orientierung

8 Gemeint sind damit alle wertgebundenen Rahmen, in welche die CDU/CSU in den Beiträgen der FAZ in dem betreffenden Wahljahr gestellt wird.

9 Der Wertebereich der Instrumentalität reichte von -1 (,Gefahr`) bis +1 (,Garant` für den jeweiligen Wert). 
der CDU/CSU von $+14,4$ in der Berichterstattung der FAZ im betreffenden Wahljahr. Wenn die FAZ die SPD ebenso häufig in diesen Wertekontext stellt, dabei aber als weitgehend hinderlich für Freiheit präsentiert $(-0,8)$, dann beträgt die Werte-Positionierung der SPD in Bezug auf Freiheit -14,4. Die durchschnittliche Werte-Positionierung für die jeweilige Partei lässt sich beispielsweise für alle Zeitungen mit vergleichbarer redaktioneller Linie (z. B. linke Zeitungen) und auch für ganze Werte-Bündel (z. B. libertäre Werte) berechnen.

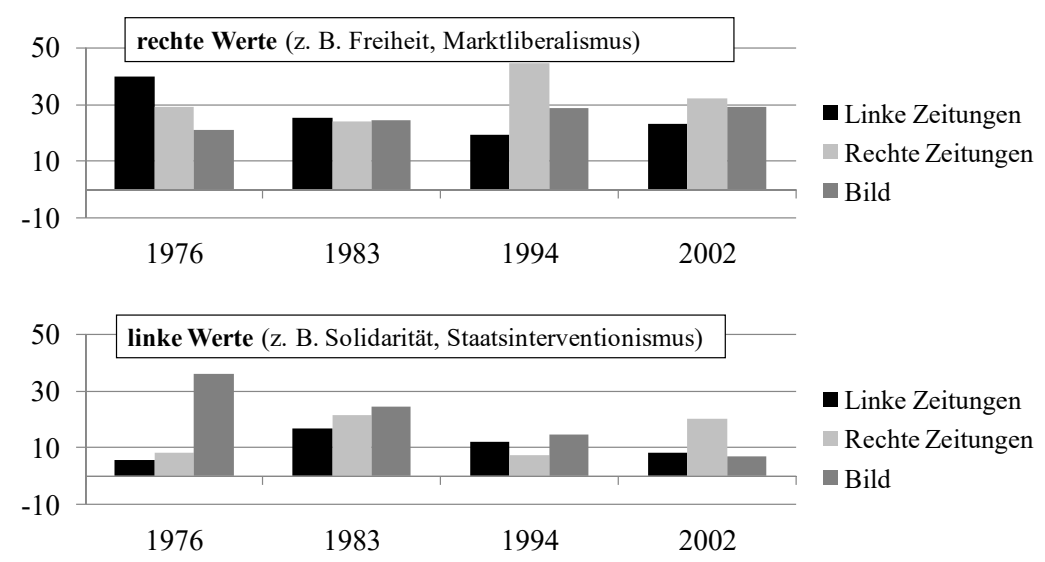

Abb. 2: Publizistische Werte-Positionierung der CDU/CSU und ihrer Kandidaten für linke und rechte Werte

Abbildung 1 zeigt die publizistische Werte-Positionierung der SPD in den vier untersuchten Wahljahren, wobei wir nach linken und rechten Zeitungen sowie Boulevardblatt differenzieren. Alle Zeitungen positionierten die SPD in den vier Wahljahren überwiegend als Garant linker Werte (vgl. Abbildung 1, untere Hälfte), aber weder eindeutig als Garant noch eindeutig als Gefahr für rechte Werte (obere Hälfte). Die Qualitätspresse zeichnete durchaus auch ein konsonantes Bild von der CDU/CSU als Garant vor allem rechter, aber auch linker Werte. Dieses Bild wurde durch die redaktionellen Linien nur akzentuiert. Allerdings wich die BILD 1976 deutlicher von der Qualitätspresse ab als in den anderen Wahljahren (vgl. Abbildung 2). Darüber hinaus lässt sich das Bild, das die Zeitungen von der Werteheimat der beiden Parteien zeichnen, auch schlüssig mit Befunden von Wahl- bzw. Parteiprogrammanalysen in den jeweiligen Wahljahren 
(vgl. z. B. Klingemann/Volkens 2002, 515-527; Pappi/Shikano 2004, 18-21) in Verbindung bringen. Diese exemplarischen Befunde (vgl. ausführlicher z. B. Scheufele/Engelmann 2013) können an dieser Stelle genügen, um die empirische Tragfähigkeit unserer Vorgehensweise zu dokumentieren.

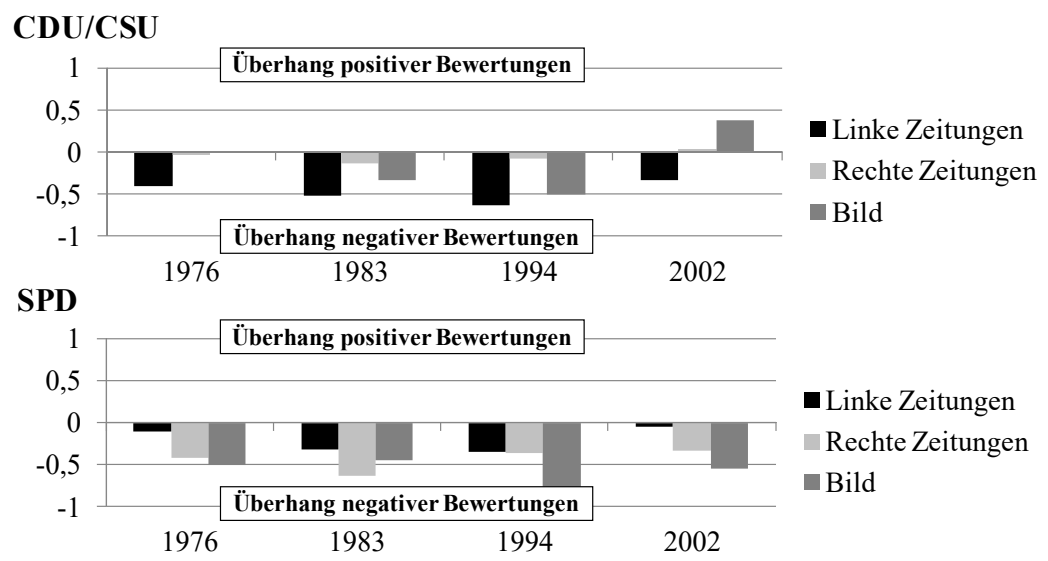

Abb. 3: Überhang positiver und negativer Bewertungen der Parteien bzw. Kandidaten - Mittelwerte (standardisiert)

Darüber hinaus können wir nicht nur theoretisch-argumentativ, sondern auch empirisch klar zwischen Frames und Bewertungen bzw. zwischen Framing und News-Bias (vgl. dazu z. B. D’Allesio/Allen 2000) unterscheiden. Denn das ValueFraming der Zeitungen war weitgehend unabhängig von den redaktionellen Linien der Zeitungen und wurde durch diese höchstens akzentuiert. Dagegen fanden wir klare Unterschiede nach politisch-redaktioneller Ausrichtung der Zeitungen in der direkten Bewertung der Parteien und deren Spitzenkandidaten (vgl. Abbildung 3). So stellten zwar auch die linken Zeitungen die SPD durchschnittlich negativ dar, aber die Union noch negativer als die SPD, wobei dieser Befund deutlicher war als bei den rechten Zeitungen. Umgekehrt bewerteten die konservativen Blätter und die BILD zwar auch die Union negativ, aber die SPD noch negativer, was wiederum deutlicher war als bei den linken Blättern. Das ist ein hinreichender empirischer Beleg für unser Argument, dass zwischen Frame und Bewertung bzw. Framing und News Bias sauber zu unterscheiden ist. 
Mediale Value-Frames - Theoretisches Konzept und methodische Herausforderungen

Unsere methodische Vorgehensweise bringt aber auch Nachteile bzw. Beschränkungen mit sich. Dazu gehören:

- Aufwand und Replizierbarkeit

- Reliabilität

- Validität

Unbestreitbar ist erstens, dass unser Vorgehen der Replizierbarkeit weit höhere Hürden setzt als z. B. einfache Themenfrequenzanalysen oder Codierungen von Frame-Elementen (z. B. Akteuren, Themen, Ursachenzuschreibungen, Maßnahmenforderungen usw.). Ebenfalls unbestreitbar ist der hohe zeitliche Aufwand sowohl für die Codierung selbst als auch für die ausführliche Schulung der Codierer, die - neben drei Reliabilitätstests - allein schon vier Wochen in Anspruch nahm. Das Kriterium der Effizienz anzulegen, ist berechtigt. Im Zweifel sollte jedoch die wissenschaftliche Erkenntnis gewichtiger sein als ein größerer Aufwand. Ob sich der Aufwand angesichts unserer Erkenntnisse gelohnt hat, müssen letztlich andere beurteilen - z. B. Drittmittelgeber oder Reviewer von wissenschaftlichen Fachzeitschriften.

Zweifellos erreicht man mit einer vergleichsweise anspruchsvollen Codierung von Value-Frames keine derart hohen Reliabilitätswerte wie bei einfachen Codierungen von Frame-Indikatoren oder Frame-Elementen. Die durchschnittliche Forscher-Codierer-Reliabilität, die an der „ratio of coding agreements to the total number of coding decisions“ (Holsti 1969, 140) bemessen wurde, lag für ValueFrames bei einer Übereinstimmung von $67 \%$ und für die Instrumentalität des Value-Framing bei $71 \%$. Solche Reliabilitätswerte sind nicht optimal, aber aus vier Gründen akzeptabel: Erstens ist der hohe Abstraktionsgrad dieser Kategorien zu beachten. Zweitens divergierten die Codierungen teilweise nicht grundsätzlich, sondern eher graduell. So erkannten die Codierer oft nicht völlig andere Werte, die wenig gemeinsam haben (z. B. Freiheit statt Solidarität), sondern unterschieden sich in der Codierung von Werten mit benachbartem semantischen Gehalt (z. B. Gleichheit statt Gerechtigkeit). Drittens werden solche graduellen NichtÜbereinstimmungen bei der Auswertung durch Bündelung von einzelnen Werten zu bestimmten Wertehorizonten und Ideologien teilweise kompensiert. Viertens war die Reliabilität keineswegs geringer als bei vergleichbaren Studien mit ähnlich aufwändiger Codierung (vgl. Eilders/Lüter 1998, 59-60; Voltmer 1997, 181). 
Bei den Beschränkungen unserer Vorgehensweise ist auch die Frage der Validität zu diskutieren: Denkbar ist erstens der Einwand, dass die Vorgabe vorab definierter Value-Frames der veränderten Bedeutung von Werten bzw. dem gesellschaftlichen Wertewandel nicht hinreichend gerecht werde. Allerdings ist der Zeitrahmen, den die vier von uns untersuchten Wahlen abstecken, im größeren zeitlichen Kontext durchaus überschaubar. Denn die epochalen Umbrüche z. B. in den Vorstellungen von Freiheit sind hier schon Geschichte. Wir wollen dennoch nicht bestreiten, dass sich das Freiheitsverständnis etwa im Zuge der 1968er Jahre oder nach dem Mauerfall verändert hat oder es diesbezüglich in manchen Kohorten noch Unterschiede zwischen Ost- und Westdeutschen geben mag. Zweitens haben wir ein breites Verständnis der jeweiligen Werte als Maßstab angelegt, weil wir ganz verschiedene Quellen für die Kategorienbildung herangezogen haben. Nuancen im Verständnis etwa von Solidarität zwischen der SPD und der Linken oder zwischen der CSU und den Grünen lassen sich mit unserem Codierschema nicht erfassen. Vermutlich bestehen die Unterschiede aber eher darin, bei welchen konkreten Problemen (z. B. Vorratsdatenspeicherung) welche Partei welchen Wert einfordert. Strittig wäre dann weniger der semantische Kern des betreffenden Frames als vielmehr das Framing, also die Frage, wann welcher Frame angewendet und mit welchen anderen Werten er kombiniert wird. Zudem muss man die Umkehrung mitdenken: Würden wir den Freiheitsbegriff nur eines einzigen Autors oder nur einer einzelnen Partei als Maßstab für unsere Codierung anlegen, dann wäre dieses Vorgehen weit weniger valide als der letztlich von uns realisierte methodische Vorschlag.

\section{Fazit}

Unseren theoretischen Überlegungen zu medialen Value-Frames liegen - wie eingangs erwähnt - eine kommunikationswissenschaftliche, eine empirische, eine problemorientierte und eine vergleichende Perspektive zugrunde. Aus diesem vierfachen Blickwinkel ist auch unser methodisches Vorgehen zur Erfassung von Value-Frames zu bewerten. Für unsere Fragestellung halten wir unsere methodische Vorgehensweise für sinnvoll und gangbar. Für andere Fragestellungen mag dies freilich anders zu bewerten sein. 
Framing hat zwar mit Deutungshoheit zu tun, ${ }^{10}$ dennoch gibt es keine Deutungshoheit über Framing oder über die Erfassung von Frames. Kein methodischer Zugang ist von vornherein besser als der andere. Vielfach stehen sich methodische Vorschläge näher als die methodologischen Lager es teilweise wahrhaben wollen. So hat Glaser $(1992,7)$ einmal zu Recht betont, dass die Grundlagen der Grounded Theory in vielem der quantitativ-explorativen Methodik (z. B. Clusteranalyse) entsprechen bzw. gleichen. Das lässt sich auch auf die denkbaren methodischen Zugänge zur Erfassung von Medien-Frames übertragen. Am sinnvollsten ist es, aus einer problemorientierten Perspektive heraus entsprechende Theorien und Modelle aufzugreifen oder zu entwickeln und danach zu klären, welche methodischen Umsetzungen passfähig sind - und nicht von vornherein nach methodologischen Präferenzen zu entscheiden. Auch unser Vorgehen ist keine Musterlösung, sondern hat Vorteile, aber auch Nachteile und Beschränkungen - wie alle anderen Vorschläge zur Erfassung von Value-Frames auch. Gleichwohl zeigt unser konzeptioneller und methodischer Vorschlag, dass die Einschätzung von Ziem $(2013,164)$ durchaus zutreffend ist, wonach es „nicht hilfreich [sei], Frame-Analysen einseitig auf die von Entman vorgeschlagenen Kategorien zu beschränken“.

\section{Literatur}

Ajzen, Icek (2005): Attitudes, personality and behavior. 2. Auflage. Berkshire: Open University Press.

Andersen, Uwe / Woyke, Wichard (Hrsg.) (2003): Handwörterbuch des politischen Systems der Bundesrepublik Deutschland. 5., aktualisierte Auflage. Opladen: Leske + Budrich.

Anderson, John R. (1996): Kognitive Psychologie. 2. Auflage. Heidelberg u. a.: Spektrum Akademischer Verlag.

Ayers, Jeffrey M. (2004): Framing collective action against neoliberalism: The case of the „anti-globalization“-movement. Journal of World-Systems Research X, $11-34$.

\footnotetext{
${ }^{10}$ Nicht nur Schwab-Trapps (2002) diskurstheoretische Arbeit zum Wandel des Kriegs- und Verteidigungsdiskurses macht deutlich, dass öffentliche Diskurse vielfach Deutungskonflikte sind. Auch Walgrave/Manssens $(2005,116)$ sprechen vom „battle“ oder "fight for the definition and framing of the covered issue".
} 
Beck, Klaus / Vowe, Gerhard (1995): Multimedia aus der Sicht der Medien. Argumentationsmuster und Sichtweisen in der medialen Konstruktion. Rundfunk \& Fernsehen 43, 549-563.

Benford, Robert D. (1997): An insider's critique of the social movement framing perspective. Sociological Inquiry 67, 409-430.

Benford, Robert D. / Snow, David A. (2000): Framing processes and social movements: An overview and assessment. Annual Review of Sociology 26, 611-639.

Brettschneider, Frank (2002): Spitzenkandidaten und Wahlerfolg. Personalisierung - Kompetenz - Parteien. Ein internationaler Vergleich. Opladen: Westdeutscher Verlag.

Brewer, Paul R. (2002): Framing, value words, and citizens' explanations of their issue opinions. Political Communication 19, 303-316.

Brewer, Paul R. / Gross, Kimberley (2005): Values, framing, and citizens' thoughts about policy issues. Effects on content and quantity. Political Psychology 26, 929-948.

Busse, Dietrich / Teubert, Wolfgang (1994): Ist Diskurs ein sprachwissenschaftliches Objekt? Zur Methodenfrage der historischen Semantik. In: Busse, Dietrich/Hermanns, Fritz/Teubert, Wolfgang (Hrsg.), Begriffsgeschichte und Diskursgeschichte. Methodenfragen und Forschungsergebnisse der historischen Semantik. Opladen: Westdeutscher Verlag, 10-28.

Cappella, Joseph N. / Jamieson, Kathleen Hall (1997): Spiral of cynicism. The press and the public good. New York, Oxford: Oxford University Press.

Dahinden, Urs (2006): Framing. Eine integrative Theorie der Massenkommunikation. Konstanz: UVK.

D’Allesio, Dave / Allen, Mike (2000): Media bias in presidential elections: A metaanalysis. Journal of Communication 50, 133-156.

De Vreese, Claes H. / Peter, Jochen / Semetko, Holli A. (2001): Framing politics at the launch of the euro: A cross-national comparative study of frames in the news. Political Communication 18, 107-122.

De Vreese, Claes H. (2010): Framing the economy. Effects of journalistic news frames. In: D’Angelo, Paul / Kuypers, Jim A. (Hrsg.), Doing news framing analy-sis: Empirical and theoretical perspectives. New York: Routledge, 187214. 
Domke, David / Shah, Dhavan D. / Wackman, Danile B. (1998): Media priming effects: Accessibility, association, and activation. International Journal of Public Opinion Research 10, 51-74.

Dulinski, Ulrike (2006): Sensationen für Millionen - das Besondere der Boulevardpresse in Deutschland. In: Ganguin, Sonja / Sander, Uwe (Hrsg.), Sensation, Skurrilität und Tabus in den Medien. Wiesbaden: VS Verlag, 23-34.

Eilders, Christiane (2004a): Von Links bis Rechts - Deutung und Meinung in Pressekommentaren. In: Eilders, Christiane / Neidhardt, Friedhelm / Pfetsch, Barbara (Hrsg.), Die Stimme der Medien. Pressekommentare und politische Öffentlichkeit in der Bundesrepublik. Wiesbaden: VS Verlag, 129-166.

Eilders, Christiane (2004b): Fokussierung und Konsonanz im Mediensystem - zu den Voraussetzungen politischer Medienwirkungen. In: Eilders, Christiane / Neidhardt, Friedhelm / Pfetsch, Barbara (Hrsg.), Die Stimme der Medien. Pressekommentare und politische Öffentlichkeit in der Bundesrepublik. Opladen: VS Verlag, 196-226.

Eilders, Christiane / Lüter, Albrecht (1998): Methodenbericht zum Projekt: Die Stimme der Medien im politischen Prozeß - Themen und Meinungen in Pressekommentaren. WZB-Discussion Paper FS III 98-107. Berlin: Wissenschaftszentrum Berlin für Sozialforschung.

Entman, Robert M. (1993): Framing: Toward clarification of a fractured paradigm. Journal of Communication 43, 51-58.

Entman, Robert M. (2007): Framing bias. Media in the distribution of power. Journal of Communication 57, 163-173.

Fair, Jo Ellen / Astroff, Roberta J. (1991): Constructing race and violence: US. news coverage and the signifying practices of Apartheid. Journal of Communication $41,58-74$.

Flanagan, Scott C. / Lee, Aie-Rie (2003): The new politics, culture wars, and the authoritarian-libertarian value change in advanced industrial democracies. Comparative Political Studies 36, 235-270.

Fishbein, Martin / Ajzen, Icek (1975): Belief, attitude, intention, and behavior: An introduction to theory and research. Reading, MA: Addison-Westley.

Fuchs, Dieter (1991): Zum Wandel politischer Konfliktlinien: Ideologische Gruppierungen und Wahlverhalten. In: Süß, Werner (Hrsg.), Die Bundesrepublik 
in den achtziger Jahren. Innenpolitik, politische Kultur, Außenpolitik. Opladen: Leske + Budrich, 69-86.

Fuchs, Dieter (2002): Das Konzept der politischen Kultur: Die Fortsetzung einer Kontroverse in konstruktiver Absicht. In: Fuchs, Dieter / Rolle, Edeltraud / Weßels, Bernhard (Hrsg.), Bürger und Demokratie in Ost und West. Studien zur politischen Kultur und zum politischen Prozess. Wiesbaden: VS Verlag, $27-49$.

Fuchs, Dieter / Klingemann, Hans D. (1989): Das Links-Rechts-Schema als politischer Code. Ein interkultureller Vergleich auf inhaltsanalytischer Grundlage. In: Haller, Max / Hoffmann-Nowotny, Hans-Joachim / Zapf, Wolfgang (Hrsg.), Kultur und Gesellschaft: Verhandlungen des 24. Deutschen Soziologentags, des 11. Österreichischen Soziologentags und des 8. Kongresses der Schweizerischen Gesellschaft für Soziologie in Zürich 1988. Frankfurt a. M., New York: Campus, 484-498.

Gabriel, Oscar W. (2009): Politische Kultur. In: Kaina, Viktoria / Römmele, Andrea (Hrsg.), Politische Soziologie. Ein Studienbuch. Wiesbaden: VS Verlag, 17-51. Gamson, William A. / Modigliani, Andre (1989): Media discourse and public opinion on nuclear power: A constructionist approach. American Journal of Sociology 95, 1-37.

Gitlin, Todd (1980): The whole world is watching: Mass media in the making \& unmaking of the new left. Berkely, CA: University of California Press.

Glaser, Barney G. (1992): Basics of grounded theory. Emergence vs. forcing. 2. Auflage. Mill Valley, CA: Sociology Press.

Goffman, Erving (1993): Rahmen-Analyse. Ein Versuch über die Organisation von Alltagserfahrungen. 3. Auflage. Frankfurt/Main: Suhrkamp.

Göhler, Gerhard / Iser, Matthias / Kerner, Ina (Hrsg.) (2006): Politische Theorie. 22 umkämpfte Begriffe zur Einführung. Unveränderter Nachdruck von 2004. Wiesbaden: VS Verlag.

Habermas, Jürgen (1995 [1981]): Theorie des kommunikativen Handelns. Band 2: Zur Kritik der funktionalistischen Vernunft. Frankfurt/Main: Suhrkamp.

Harden, Lars (2002). Rahmen der Orientierung. Eine Längsschnittanalyse von Frames in der Philosophieberichterstattung deutscher Qualitätsmedien. Wiesbaden: Deutscher Universitätsverlag. 
Hildebrandt, Kai / Dalton, Russell J. (1977): Politischer Wandel oder Schönwetterpolitik? Politische Vierteljahresschrift 18, 230-256.

Holsti, Ole R. (1969): Content analysis for the social sciences and humanities. Reading, MA: Addison-Wesley.

Holtmann, Everhard / Brinkmann, Heinz Ulrich / Pehle, Heinrich (Hrsg.) (1991): Politik-Lexikon. München, Wien: R. Oldenbourg.

Inglehart, Ronald (1977): The silent revolution: Changing values and political styles among Western publics. Princeton, NY: Princeton University Press.

Inglehart, Ronald (1988): Modernisierung und Postmodernisierung. Kultureller, wirtschaftlicher und politischer Wandel in 43 Gesellschaften. Frankfurt/Main, New York: Campus.

Iyengar, Shanto (1991): Is anyone responsible? How television frames political issues. Chicago: The University of Chicago Press.

Johnston, Hank / Noakes, John A. (Hrsg.) (2005): Frames of protest: Social movements and the framing perspective. Lanham, MD: Rowman \& Littlefield Publishers.

Klein, Markus (2005): Gesellschaftliche Wertorientierungen, Wertewandel und Wählerverhalten. In: Falter, Jürgen W. / Schoen, Harald (Hrsg.), Handbuch Wahlforschung. Wiesbaden: VS Verlag, 423-445.

Klingemann, Hans-Dieter (1989): Die programmatischen Profile der politischen Parteien in der Bundesrepublik Deutschland. Eine quantitative Inhaltsanalyse der Wahlprogramme von SPD, FDP und CDU von 1949 bis 1987. In: Herzog, Dietrich/Weßels, Bernhard (Hrsg.), Konfliktpotentiale und Konsensstrategien. Beiträge zur politischen Soziologie der Bundesrepublik (Schriften des Zentralinstituts für sozialwissenschaftliche Forschung der FU Berlin; Bd. 54). Opladen: Westdeutscher Verlag, 99-115.

Klingemann, Hans-Dieter / Volkens, Andrea (2002): Struktur und Entwicklung von Wahlprogrammen in der Bundesrepublik Deutschland 1949-1998. In: Gabriel, Oscar W. / Niedermayer, Oskar / Stöss, Richard (Hrsg.), Parteiendemokratie in Deutschland. 2., aktualisierte und erweiterte Auflage. Wiesbaden: Westdeutscher Verlag, 507-527.

Lieske, Sandra (2005): Die Anzeigenkampagne zur Europawahl 2004. In: HoltzBacha, Christina (Hrsg.), Europawahl 2004. Die Massenmedien im Europawahlkampf. Wiesbaden: VS Verlag, 90-122. 
Lipset, Seymour Martin / Rokkan, Stein (1967): Cleavage structures, party systems, and voter alignments: An introduction. In: Lipset, Seymour Martin / Rokkan, Stein (Hrsg.), Party systems and voter alignments: Cross-national perspectives. New York: The Free Press, 1-64.

Mahrt, Merja (2010): Values of german media users: 1986-2007. Wiesbaden: VS Verlag.

Matthes, Jörg (2007): Framing-Effekte. Zum Einfluss der Politikberichterstattung auf die Einstellungen der Rezipienten (Rezeptionsforschung Bd. 13). München: R. Fischer.

Matthes, Jörg / Kohring, Mathias (2004): Die empirische Erfassung von MedienFrames. Medien \& Kommunikationswissenschaft 52, 56-75.

Mayring, Philipp (2000): Qualitative Inhaltsanalyse. Grundlagen und Techniken. 7. Auflage. Weinheim.

Minsky, Marvin (1974): A framework for representative knowledge. Artificial Knowledge Memo No. 396. Boston: MIT.

Merton, Robert K. (1996): On social structure and science (edited and with an introduction by Sztompka, Piotr) Chicago: University of Chicago Press.

Nelson, Thomas E. / Oxley, Zoe M. / Clawson, Rosalee A. (1997a): Toward a psychology of framing effects. Political Behavior 19, 221-246.

Nelson, Thomas E. / Clawson, Rosalee A. / Oxley, Zoe M. (1997b): Media framing of civil liberties conflict and its effects on tolerance. American Political Science Review 91, 567-583.

Neuman, W. Russell / Just, Marion R. / Crigler, Ann N. (1992): Common knowledge. News and the construction of political meaning. Chicago, London: The University of Chicago Press.

Niedermayer, Oskar (2003): Die Entwicklung des deutschen Parteiensystems bis nach der Bundestagswahl 2002. In: Niedermayer, Oskar (Hrsg.), Die Parteien nach der Bundestagswahl 2002. Opladen: Leske + Budrich, 9-41.

Nohlen, Dieter / Schultze, Rainer-Olaf (Hrsg.) (2005a,b): Lexikon der Politikwissenschaft. Theorien. Methoden, Begriffe. Band 1: A-M; Band 2: N-Z. Aktualisierte und erweiterte Auflage. München: C. H. Beck.

Opp, Karl-Dieter (1970): Theories of the middle range as a strategy for the construction of a general sociological theory. A critique of a sociological dogma. Quality \& Quantity 4, 243-253. 
Pappi, Franz Urban / Shikano, Susumu (2002): Die politisierte Sozialstruktur als mittelfristig stabile Basis einer deutschen Normalwahl. Kölner Zeitschrift für Soziologie und Sozialpsychologie 54, 444-475.

Pappi, Franz Urban / Shikano, Susumu (2004): Ideologische Signale in den Wahlprogrammen der deutschen Bundestagsparteien 1990 bis 2002. Working-Paper Nr. 76. Mannheim: Mannheimer Zentrum für Europäische Sozialforschung.

Parsons, Talcott (1951): The social system. New York: The Free Press.

Price, Vincent / Tewksbury, David (1997): News Values and Public Opinion. A Theoretical Account of Media Priming and Framing. In: Barett, George A. I Boster, Franklin J. (Hrsg.), Progress in Communication Sciences. Advances in Persuasion. Vol. 13. Greenwich, CT: Ablex, 173-212.

Reinemann, Carsten (2003): Medienmacher als Mediennutzer. Kommunikationsund Einflussstrukturen im politischen Journalismus der Gegenwart (Medien in Geschichte und Gegenwart; Bd. 19). Köln, Weimar, Wien: Böhlau.

Reinemann, Carsten (2008): „Guter Boulevard ist immer auch außerparlamentarische Opposition“ - Das Handeln von Bild am Beispiel der Berichterstattung über Hartz IV. In: Pfetsch, Barbara / Adam, Silke (Hrsg.), Massenmedien als politische Akteure. Konzepte und Analysen. Wiesbaden: VS Verlag, 196-224.

Roller, Edeltraud (2000): Marktwirtschaftliche und wohlfahrtstaatliche Gerechtigkeitsprinzipien in Deutschland und den USA. In: Gerhards, Jürgen (Hrsg.), Die Vermessung kultureller Unterschiede: USA und Deutschland im Vergleich. Wiesbaden: Westdeutscher Verlag, 89-110.

Roller, Edeltraud (2002): Erosion des sozialstaatlichen Konsenses und die Entstehung einer neuen Konfliktlinie in Deutschland. Aus Politik und Zeitgeschichte, Beilage zur Wochenzeitschrift Das Parlament, B 29-30/2002, 13-19.

Rosenberg, Milton J. (1956): Cognitive structure and attitudinal affect. Journal of Abnormal and Social Psychology 53, 367-372.

Schank, Roger C. / Abelson, Robert P. (1977): Scripts, plans, goals, and understanding: An inquiry into human knowledge structures. Hillsdale, NJ: Lawrence Erlbaum.

Scheufele, Bertram (2004): Framing-effects approach: A theoretical and methodological critique. Communications 29, 401-428. 
Scheufele, Bertram (2010): Verknüpfen und Urteilen. Ein Experiment zur Wirkung medialer Value-Frames. Medien \& Kommunikationswissenschaft 58, 26-45.

Scheufele, Bertram / Engelmann, Ines (2012): Value-Framing. Mediendarstellung und Medienwirkung bei ausgewählten Bundestagswahlen. Abschlussbericht zum DFG-Projekt SCHE 697/6-1. Stuttgart: Universität Hohenheim.

Scheufele, Bertram / Engelmann, Ines (2013): Die publizistische Vermittlung von Wertehorizonten der Parteien. Normatives Modell und empirische Befunde zum Value-Framing und News Bias der Qualitäts- und Boulevardpresse bei vier Bundestagswahlen. Medien \& Kommunikationswissenschaft 61, 532-550.

Scheufele, Bertram / Scheufele, Dietram A. (2010): Of spreading activation, applicability and schemas: Conceptual distinctions and their operational implications for measuring frames and framing effects. In: D’Angelo, Paul / Kuypers, Jim A. (Hrsg.), Doing news framing analysis: Empirical and theoretical perspectives. New York: Routledge, 110-134.

Scheufele, Dietram A. (1999): Framing as a theory of media effects. Journal of Communication 49(1), 103-122.

Schirmer, Stefan (2001): Die Titelseitenaufmacher der Bild-Zeitung im Wandel. Eine Inhaltsanalyse unter Berücksichtigung von Merkmalen journalistischer Qualität (Reihe Medienskripten; Bd. 35). München: R. Fischer.

Schubert, Klaus / Klein, Martina (2006): Das Politiklexikon. 4., erweiterte und aktualisierte Auflage. Bonn: Dietz.

Schulz, Winfried (2011): Politische Kommunikation. Theoretische Ansätze und Ergebnisse empirischer Forschung. 3., überarbeitete Auflage. Wiesbaden: VS Verlag.

Schwab-Trapp, Michael (2002). Kriegsdiskurse. Die politische Kultur des Krieges 1991 bis 1999. Opladen: Leske + Budrich.

Schwartz, Shalom H. / Bardi, Anat (2001): Value hierarchies across cultures. Taking a similarities perspective. Journal of Cross-Cultural Psychology 32, 268-290.

Semetko, Holli A. / Schoenbach, Klaus (2003): News and elections. German Bundestag campaigns in the Bild, 1990-2002. The International Journal of Press/Politics 8(3), 54-69. 
Semetko, Holly A. / Valkenburg, Patti M. (2000): Framing European politics: A content analysis of press and television news. Journal of Communication 50, 93-109.

Shah, Dhavan V. / Domke, David / Wackman, Daniel B. (1996): „To thine own self be true“. Values, framing, and voter decision-making strategies. Communication Research 23, 509-560.

Shah, Dhavan V. / Domke, David / Wackman, Daniel B. (2001): The effects of value-framing on political judgement and reasoning. In: Reese, Stephen D. I Gandy, Oscar H. / Grant, August E. (Hrsg.), Framing public life. Perspectives on media and our understanding of the social world. Mahwah, N J, London: Lawrence Erlbaum, 227-243.

Sheafer, Tamir / Weimann, Gabriel (2005): Agenda building, agenda setting, priming, individual voting intentions, and the aggregate results. An analysis of four Israeli elections. Journal of Communication 55, 347-365.

Shen, Fuyuan / Edwards, Heidi Hatfield (2005): Economic individualism, humanitarianism, and welfare reform. A value-based account of framing effects. Journal of Communication 55, 795-809.

Snow, David A. / Benford, Robert D. (1988): Ideology, frame resonance, and participant mobilization. In: Klandermans, Bert / Kriesi, Hanspeter / Tarrow, Sidney (Hrsg.), International social movement, Vol. 1: From structure to action: Comparing social movement research across cultures. Greenwich, CT: JAI Press, 197-217.

Snow, David A. / Benford, Robert D. (1992): Master frames and cycles of protest. In: Morris, Aldon D. / McClurg Mueller, Carol (Hrsg.), Frontiers in social movement theory. New Haven, CT: Yale University Press, 133-155.

Stöss, Richard (1997): Stabilität im Umbruch. Wahlbeständigkeit und Parteienwettbewerb im ,Superwahljahr' 1994. Opladen: Westdeutscher Verlag.

Tankard, James W. (2001): The empirical approach to the study of framing. In: Reese, Stephen D. / Gandy, Oscar H. / Grant, August E. (Hrsg.): Framing public life. Perspectives on media and our understanding of the social world. Mahwah (NJ)/London: Lawrence Erlbaum, 95-106.

Tesser, Abraham (1978): Self-generated attitude change, in: Berkowitz, Leonard (Hrsg.), Advances in experimental social psychology. Volume 11. New York u. a.: Academic Press, 289-233. 
Tversky, Amos / Kahneman, Daniel (1986): Rational Choice and the framing of decisions. Journal of Business 19, 251-278.

Valkenburg, Patti M. / Semetko, Holly A. / de Vreese, Claes H. (1999): The effects of news frames on readers's thoughts and recall. Communication Research 26, 550-569.

Verba, Sidney (1965): Comparative political culture. In: Pye, Lucian W. / Verba, Sidney (Hrsg.), Political culture and political development. Princeton, N J: Princeton University Press, 512-560.

Vlasic, Andreas (2004): Die Integrationsfunktion der Massenmedien. Begriffsgeschichte, Modelle, Operationalisierung. Wiesbaden: VS Verlag.

Volkens, Andrea (1996): Parteiprogramme und Polarisierung. In: Niedermayer, Oskar (Hrsg.), Intermediäre Strukturen in Ostdeutschland (Beiträge zu den Berichten der Kommission für die Erforschung des sozialen und politischen Wandels in den neuen Bundesländern e.V.; Bd. 3.2). Opladen: Leske + Budrich, 215-236.

Volkens, Andrea (2002): Handbuch zur Inhaltsanalyse programmatischer Dokumente von Parteien und Regierungen in der Bundesrepublik Deutschland. WZB-Discussion Paper FS III 02-203. Berlin: Wissenschaftszentrum Berlin für Sozialforschung.

Voltmer, Katrin (1997): Medien und Parteien im Wahlkampf. Die ideologischen Präferenzen der meinungsführenden Tageszeitungen im Bundestagswahlkampf 1990. Rundfunk \& Fernsehen 45, 173-197.

Walgrave, Stefaan / Manssens, Jan (2005): Mobilizing the white march: media frames as alternatives to movement organizations. In: Johnston, Hank / Noakes, John A. (Hrsg.), Frames of protest. Social movements and the framing perspective. Lanham, MD: Rowman \& Littlefield Publishers, 113-140.

Wilke, Jürgen (1999): Leitmedien und Zielgruppenorgane. In: Wilke, Jürgen (Hrsg.), Mediengeschichte der Bundesrepublik Deutschland. Köln, Weimar, Wien: Böhlau, 302-329.

Wilke, Jürgen / Reinemann, Carsten (2000): Kanzlerkandidaten in der Wahlberichterstattung 1949-1998. Köln, Weimar, Wien: Böhlau.

Wüst, Andreas M. / Volkens, Andrea (2003): Euromanifesto coding instructions. Working Paper Nr. 64. Mannheim: Mannheimer Zentrum für Europäische Sozialforschung. 
Zeller, Frauke / Wolling, Jens / Porten-Cheé, Pablo (2010): Framing 0/1. Wie die Medien über die „Digitalisierung der Gesellschaft“ berichten. Medien \& Kommunikationswissenschaft 58, 503-524.

Ziem, Alexander (2008): Frames und sprachliches Wissen. Kognitive Aspekte der semantischen Kompetenz (Sprache und Wissen; Bd. 2). Berlin/New York: de Gruyter.

Ziem, Alexander (2013): Frames als Prädiktions- und Medienrahmen: Auf dem Weg zu einem integrativen Ansatz? In: Fraas, Claudia / Meier, Stefan / Pentzold, Christian (Hrsg.), Online-Diskurse. Theorien und Methoden transmedialer Online-Diskursforschung. Köln: Herbert von Halem, 136-172.

Ziem, Alexander (2014): Von der Kasusgrammatik zum FrameNet: Frames, Konstruktionen und die Idee eines Konstruktikons. In: Ziem, Alexander / Lasch, Alexander (Hrsg.), Grammatik als Netzwerk von Konstruktionen? Sprachwissen im Fokus in der Konstruktionsgrammatik (Sprache und Wissen; Bd. 15). Berlin: de Gruyter, 263-290. 
